\title{
THE FOURIER TRANSFORM OF ORDER STATISTICS WITH APPLICATIONS TO LORENTZ SPACES
}

\author{
S. J. Dilworth And A. L. Koldobsky
}

\begin{abstract}
We present a formula for the Fourier transforms of order statistics in $\mathbb{R}^{n}$ showing that all these Fourier transforms are equal up to a constant multiple outside the coordinate planes in $\mathbb{R}^{n}$.

For $a_{1} \geq \ldots \geq a_{n} \geq 0$ and $q>0$, denote by $\ell_{w, q}^{n}$ the $n$-dimensional Lorentz space with the norm $\left\|\left(x_{1}, \ldots, x_{n}\right)\right\|=\left(a_{1}\left(x_{1}^{*}\right)^{q}+\ldots+a_{n}\left(x_{n}^{*}\right)^{q}\right)^{1 / q}$, where $\left(x_{1}^{*}, \ldots, x_{n}^{*}\right)$ is the non-increasing permutation of the numbers $\left|x_{1}\right|, \ldots,\left|x_{n}\right|$. We use the above mentioned formula and the Fourier transform criterion of isometric embeddability of Banach spaces into $L_{q}$ [10] to prove that, for $n \geq 3$ and $q \leq 1$, the space $\ell_{w, q}^{n}$ is isometric to a subspace of $L_{q}$ if and only if the numbers $a_{1}, \ldots, a_{n}$ form an arithmetic progression. For $q>1$, all the numbers $a_{i}$ must be equal so that $\ell_{w, q}^{n}=\ell_{q}^{n}$. Consequently, the Lorentz function space $L_{w, q}(0,1)$ is isometric to a subspace of $L_{q}$ if and only if either $0<q<\infty$ and the weight $w$ is a constant function (so that $L_{w, q}=L_{q}$ ), or $q \leq 1$ and $w(t)$ is a decreasing linear function.
\end{abstract}

Finally, we relate our results to the theory of positive definite functions.

\section{INTRODUCTION}

For a vector $x=\left(x_{1}, \ldots, x_{n}\right) \in \mathbb{R}^{n}$, denote by $x^{*}=\left(x_{1}^{*}, \ldots, x_{n}^{*}\right)$ the non-increasing permutation of the numbers $\left|x_{1}\right|, \ldots,\left|x_{n}\right|$. We shall consider the order statistics $x_{k}^{*}$ as functions of the variables $x_{1}, \ldots, x_{n}$.

For $a_{1} \geq \ldots \geq a_{n} \geq 0$ (not all zero) and $q>0$, the expression $\left\|\left(x_{1}, \ldots, x_{n}\right)\right\|=$ $\left(a_{1}\left(x_{1}^{*}\right)^{q}+\ldots+a_{n}\left(x_{n}^{*}\right)^{q}\right)^{1 / q}$ is the norm (or $q$-norm if $q<1$ ) of an $n$-dimensional weighted Lorentz space which, as usual, we denote by $\ell_{w, q}^{n}$. For an infinite decreasing sequence $w=\left\langle a_{n}\right\rangle$ of positive weights, for which $\sum_{n=1}^{\infty} a_{n}=\infty$, the Lorentz sequence space $\ell_{w, q}$ is defined similarly.

Let $I$ denote the interval $(0,1)$ or the interval $(0, \infty)$, and let $w(t)$ be a positive decreasing function defined on $I$ for which $\int_{0}^{1} w(t) d t=1$ and $\int_{0}^{\infty} w(t) d t=\infty$ (the latter condition only for $I=(0, \infty))$. For $0<q<\infty$, the Lorentz function space $L_{w, q}(I)$ is the space of equivalence classes of real-valued measurable functions $f$ on

1991 Mathematics Subject Classification. Primary 46B04. Secondary 42B10, 46E30, 52B12, 60E10, 62G30.

Key words and phrases. Fourier transform, isometry, Lorentz spaces, order statistics.

Both authors were supported in part by the NSF Workshop in Linear Analysis and Probability held at Texas A\&M University in August 1993 
$I$ for which the following norm (or $q$-norm if $q<1$ )

$$
\|f\|_{w, q}=\left(\int_{I} f^{*}(t)^{q} w(t) d t\right)^{\frac{1}{q}}
$$

is finite, where $f^{*}$ denotes the non-increasing rearrangement of $|f|$. For $0<q \leq p<$ $\infty$, the classical Lorentz spaces $L_{p, q}$ introduced in [15] correspond to the weights $w(t)=(q / p) t^{\frac{q}{p}-1}$.

Schütt [19] proved that, if $1 \leq q<2$, then $L_{w, q}(0,1)$ is isomorphic to a subspace of $L_{q}$ if and only if it is a $q$-concave Banach lattice. For the classical spaces $L_{p, q}$, it follows from Schütt's result, from Carothers and Dilworth [4], and from M. Levy $[12,13]$, that $L_{p, q}$ is isomorphic to a subspace of $L_{q}$ if and only if $p=q$ or $0<$ $q \leq p<2$. For further isomorphic results about the subspace structure of the $L_{p, q}$ spaces we refer the reader to $[3,8,12,13]$.

The initial purpose of this work was to check the isometric version of the above results. We started with the question of whether any finite-dimensional Lorentz space $\ell_{w, q}^{n}$ is isometric to a subspace of $L_{q}$ ? Since we expected a negative answer for $n \geq 3$, we were going to use the following Fourier transform criterion from [10]: if $q>0$, where $q$ is not an even integer, and if the $n$-dimensional quasi-Banach space $E$ is isometric to a subspace of $L_{q}$, then the distribution

$$
\gamma\left(\xi_{1}, \ldots, \xi_{n-1}\right)=\frac{1}{(2 \pi)^{n-1} c_{q}}\left(\|x\|^{q}\right)^{\wedge}\left(\xi_{1}, \ldots, \xi_{n-1}, 1\right)
$$

is a finite measure on $\mathbb{R}^{n-1}$ (here $c_{q}=2^{q+1} \pi^{1 / 2} \Gamma((q+1) / 2) / \Gamma(-q / 2)$, and the Fourier transform is considered in the sense of distributions).

Calculating the Fourier transform of the norm of the space $\ell_{w, q}^{n}$ we ran into a surprising fact: for every continuous function $f$ on $\mathbb{R}$ with a power growth at infinity, all the order statistics $\sum_{k=1}^{n} a_{k} f\left(x_{k}^{*}\right)$ have equal Fourier transforms up to a constant multiple outside the coordinate planes in $\mathbb{R}^{n}$. The same result is true if we consider non-increasing permutations of $x_{1}, \ldots, x_{n}$ instead of $\left|x_{1}\right|, \ldots,\left|x_{n}\right|$. This fact has, however, a simple explanation which we present in Section 2.

In Section 3 we apply the formula for the Fourier transform of order statistics to the Lorentz sequence spaces. We prove that, for $q \leq 1$, the space $\ell_{w, q}^{n}$ is isometric to a subspace of $L_{q}$ if and only if the numbers $a_{1}, \ldots, a_{n}$ form an arithmetic progression. For $q>1$, all the numbers $a_{i}$ must be equal so that $\ell_{w, q}^{n}=\ell_{q}^{n}$.

In Section 4 we deduce from the finite-dimensional results that there are nontrivial isometric embeddings of the function space space $L_{w, q}(I)$ into $L_{q}$ if and only if $I=(0,1), q \leq 1$, and $w(t)$ is a decreasing linear function. As a consequence, we obtain an interesting family of rearrangement-invariant renormings of $L_{1}$ which are at the same time isometric to subspaces of $L_{1}$.

In Section 5 we show how our results are related to a problem of I. J. Schoenberg 
Finally, we wish to mention that the isometries of $L_{p, 1}$ into itself were determined by Carothers and Turett [6], and that recently Carothers, Haydon, and Pei-Kee Lin [7] determined the isometries of $L_{w, q}$ into itself. The methods required to prove these results are quite different from those used in this paper.

\section{The Fourier Transform of Order Statistics}

We start with the following elementary fact.

Lemma 1. For any $a_{1}, a_{2}, \ldots, a_{n} \in \mathbb{R}$, any function $f$ on $\mathbb{R}$, and every $x=$ $\left(x_{1}, \ldots, x_{n}\right) \in \mathbb{R}^{n}$, we have

$$
\begin{aligned}
a_{1} f\left(x_{1}^{*}\right) & +\cdots+a_{n} f\left(x_{n}^{*}\right) \\
& =\sum_{k=1}^{n}\left(\sum_{j=1}^{k}(-1)^{j-1}\left(\begin{array}{c}
k-1 \\
j-1
\end{array}\right) a_{n-k+j}\right) \sum_{i_{1}<\cdots<i_{k}} f\left(\max \left(\left|x_{i_{1}}\right|, \ldots,\left|x_{i_{k}}\right|\right)\right) .
\end{aligned}
$$

where the latter sum is taken over all choices of $1 \leq i_{1}<\cdots<i_{k} \leq n$.

Proof. We argue by induction. Assume (without loss of generality) that $\left|x_{1}\right| \geq\left|x_{k}\right|$ for $k=2, \ldots, n$, and suppose that our statement is true for the numbers $a_{2}, \ldots, a_{n}$ and $x_{2}, \ldots, x_{n}$. If we add $a_{1}$ and $x_{1}$ then the left-hand side of (1) changes by $a_{1} f\left(\left|x_{1}\right|\right)$. The additional summands in the right-hand side are as follows:

$$
a_{m}\left(\sum_{j=0}^{m-1}(-1)^{j}\left(\begin{array}{c}
n-m+j \\
j
\end{array}\right)\left(\begin{array}{c}
n-1 \\
n-m+j
\end{array}\right)\right) f\left(\left|x_{1}\right|\right), \quad m=1, \ldots, n .
$$

For every $m \geq 2$, the sum in parentheses is equal to

$$
\frac{(n-1) \cdot \ldots \cdot m}{(n-m) !} \sum_{j=0}^{m-1}(-1)^{j}\left(\begin{array}{c}
m-1 \\
j
\end{array}\right)=\frac{(n-1) \cdot \ldots \cdot m}{(n-m) !}((-1)+1)^{m-1}=0
$$

So the only non-zero additional summand in the right-hand side is $a_{1} f\left(\left|x_{1}\right|\right)$, which completes the proof.

As usual, we denote by $\mathcal{S}\left(\mathbb{R}^{n}\right)$ the space of rapidly decreasing infinitely differentiable functions on $\mathbb{R}^{n}$, and by $\mathcal{S}^{\prime}\left(\mathbb{R}^{n}\right)$ the space of tempered distributions. For an open subset $\Omega$ of $\mathbb{R}^{n}, \mathcal{D}(\Omega)$ denotes the collection of functions in $\mathcal{S}\left(\mathbb{R}^{n}\right)$ with compact supports in $\Omega$. We say that two distributions $f, g \in \mathcal{S}^{\prime}\left(\mathbb{R}^{n}\right)$ are equal on $\Omega$ if $\langle f, \phi\rangle=\langle g, \phi\rangle$ for every $\phi \in \mathcal{D}(\Omega)$.

The Fourier transform of any distribution in $\mathcal{S}^{\prime}\left(\mathbb{R}^{n}\right)$ of the form $g\left(x_{i_{1}}, \ldots, x_{i_{k}}\right)$, where $k<n$, is equal to zero outside the coordinate planes in $\mathbb{R}^{n}$. Using this fact 
Proposition 1. For any continuous function $f$ on $\mathbb{R}$ with power growth at infinity (i.e., for some $A>0, \rho>0,|f(x)| \leq A\left(1+|x|^{\rho}\right)$, for all $x \in \mathbb{R}$ ) and any numbers $a_{1}, a_{2}, \ldots, a_{n} \in \mathbb{R}$ the Fourier transforms of the distributions $\sum a_{k} f\left(x_{k}^{*}\right)$ and $c f\left(\max \left(\left|x_{1}\right|, \ldots,\left|x_{n}\right|\right)\right)$, where $c=\sum_{k=1}^{n}(-1)^{k-1}\left(\begin{array}{l}n-1 \\ k-1\end{array}\right) a_{k}$ are equal outside the coordinate planes in $\mathbb{R}^{n}$.

Lemma 1 also shows that the Fourier transform of order statistics can easily be calculated if we have a formula for the Fourier transform of the distributions of the form $f\left(\max \left(\left|x_{1}\right|, \ldots,\left|x_{n}\right|\right)\right)$. Such a formula was obtained in [11] in connection with some problems concerning the characterization of measures by potentials. We repeat this calculation here because the formula is crucial for our further considerations.

Denote by $G$ the set of vectors $\xi=\left(\xi_{1}, \ldots, \xi_{n}\right) \in \mathbb{R}^{n}$ such that $\xi_{k} \neq 0$ for $1 \leq k \leq n$ and $(\delta, \xi) \neq 0$ for every vector $\delta=\left(\delta_{1}, \ldots, \delta_{n}\right)$, with $\delta_{k}= \pm 1$ for $1 \leq k \leq n$ (here $(\delta, \xi)$ denotes the usual scalar product in $\mathbb{R}^{n}$ ).

Proposition 2. Let $f$ be an even continuous function on $\mathbb{R}^{n}$ with power growth at infinity and for which the distribution $u=\left(f(t)(\operatorname{sgn}(t))^{n-1}\right)^{\wedge}$ is a continuous function on $\mathbb{R} \backslash\{0\}$. Then, for every $\xi \in G$, we have

$$
\begin{aligned}
& f\left(\max \left(\left|x_{1}\right|, \ldots,\left|x_{n}\right|\right)\right)^{\wedge}(\xi) \\
& \quad=\frac{i^{n-1}}{2 \xi_{1} \cdot \ldots \cdot \xi_{n}} \sum_{\delta} \delta_{1} \cdot \ldots \cdot \delta_{n}\left(\delta_{1} \xi_{1}+\cdots+\delta_{n} \xi_{n}\right) u\left(\delta_{1} \xi_{1}+\cdots+\delta_{n} \xi_{n}\right),
\end{aligned}
$$

where the sum is taken over all changes of signs.

Proof. Let $\phi \in \mathcal{S}\left(\mathbb{R}^{n}\right)$ be a function with a compact support outside the coordinate planes. Then there exists a function $F \in \mathcal{S}\left(\mathbb{R}^{n}\right)$ such that $\partial^{n} F / \partial x_{1} \ldots \partial x_{n}=\widehat{\phi}$. To see this, note that $\psi(x)=\phi(x) /\left(x_{1} \ldots x_{n}\right)$ belongs to $\mathcal{S}\left(\mathbb{R}^{n}\right)$, and hence $\widehat{\psi} \in \mathcal{S}\left(\mathbb{R}^{n}\right)$. Put $F=i^{n} \widehat{\psi}$ and now use the connection between the Fourier transform and differentiation:

$$
\partial^{n} F / \partial x_{1} \ldots \partial x_{n}=i^{n} \partial^{n} \widehat{\psi} / \partial x_{1} \ldots \partial x_{n}=\left(x_{1} \ldots x_{n} \psi\right)^{\wedge}=\widehat{\phi}
$$

Denote by $B_{t}$ the ball $\left\{x \in \mathbb{R}^{n}:\|x\|_{\infty}<t\right\}$. Recall that, for every $\phi \in \mathcal{S}\left(\mathbb{R}^{n}\right)$ and for every non-zero $\xi \in \mathbb{R}^{n}$, the function $t \rightarrow \widehat{\phi}(t \xi)(t \in \mathbb{R})$ is the Fourier transform of the function $y \rightarrow \int_{(\xi, x)=y} \phi(x) d x(y \in \mathbb{R})$ : this is the well-known connection between the Fourier transform and the Radon transform (see e.g. [9]). Now we can 


$$
\begin{aligned}
\left\langle f\left(\max \left(\left|x_{1}\right|, \ldots,\left|x_{n}\right|\right)\right)^{\wedge}, \phi\right\rangle & =\left\langle f\left(\max \left(\left|x_{1}\right|, \ldots,\left|x_{n}\right|\right)\right), \widehat{\phi}\right\rangle \\
& =\int_{R^{n}} f\left(\max \left(\left|x_{1}\right|, \ldots,\left|x_{n}\right|\right)\right) \widehat{\phi}(x) d x \\
& =\int_{0}^{\infty} f(t)\left(\int_{B_{t}} \widehat{\phi}(x) d x\right)_{t}^{\prime} d t \\
& =\int_{0}^{\infty} f(t)\left(\int_{-t}^{t} \ldots \int_{-t}^{t} \widehat{\phi}(x) d x\right)_{t}^{\prime} d t \\
& =\int_{0}^{\infty} f(t) \sum_{\delta} \delta_{1} \cdot \ldots \cdot \delta_{n}\left(F\left(\delta_{1} t, \ldots, \delta_{n} t\right)\right)_{t}^{\prime} d t
\end{aligned}
$$

which equals

$$
\int_{0}^{\infty} f(t)\left(\sum_{\delta} \delta_{1} \cdot \ldots \cdot \delta_{n}\left(\delta_{1} \frac{\partial F}{\partial x_{1}}+\cdots+\delta_{n} \frac{\partial F}{\partial x_{n}}\right)\left(\delta_{1} t, \ldots, \delta_{n} t\right)\right) d t
$$

The function $\left(\int_{B_{t}} \widehat{\phi}(x) d x\right)_{t}^{\prime}$ is even if $n$ is an odd integer and odd if $n$ is even. Therefore the integral in (3) is equal to

$$
\frac{1}{2}\left\langle f(t)(\operatorname{sgn}(t))^{n-1}, \sum_{\delta} \delta_{1} \cdot \ldots \cdot \delta_{n}\left(\delta_{1} \frac{\partial F}{\partial x_{1}}+\cdots+\delta_{n} \frac{\partial F}{\partial x_{n}}\right)\left(\delta_{1} t, \ldots, \delta_{n} t\right)\right\rangle .
$$

Since $\partial F / \partial x_{k}=i^{n-1}\left(x_{k} \phi(x) /\left(x_{1} \ldots x_{n}\right)\right)^{\wedge}$ for each $k$, we can use the property of the Radon transform which was mentioned above to rewrite (4) as the following:

$$
\frac{i^{n-1}}{2}\left\langle f(t)(\operatorname{sgn}(t))^{n-1}, \sum_{\delta} \delta_{1} \cdot \ldots \cdot \delta_{n}\left(\sum_{j=1}^{n} \int_{(\delta, x)=y} \frac{\delta_{j} x_{j} \phi(x)}{x_{1} \ldots x_{n}} d x\right)^{\wedge}\right\rangle
$$

which equals

$$
\frac{i^{n-1}}{2}\left\langle\left(f(t)(\operatorname{sgn}(t))^{n-1}\right)^{\wedge}(y), y \sum_{\delta} \delta_{1} \cdot \ldots \cdot \delta_{n} \int_{(\delta, x)=y} \frac{\phi(x)}{x_{1} \ldots x_{n}} d x\right\rangle .
$$

The distribution $u=\left(f(t)(\operatorname{sgn}(t))^{n-1}\right)^{\wedge}$ is a continuous function on $\mathbb{R} \backslash\{0\}$, and so, for every $\phi \in \mathcal{S}\left(\mathbb{R}^{n}\right)$ with compact support in $G$, we have

$$
\left\langle f\left(\|x\|_{\infty}\right)^{\wedge}, \phi\right\rangle=\frac{i^{n-1}}{2} \int_{\mathbb{R}}\left(\sum_{\delta} \delta_{1} \ldots \cdot \delta_{n} \int_{(\delta, x)=y} \frac{\phi(x)}{x_{1} \ldots x_{n}} d x\right) y u(y) d y
$$

The latter integral converges absolutely because all the functions

$$
y \rightarrow \int \quad \frac{\phi(x)}{\infty} d x \quad(y \in \mathbb{R})
$$


belong to $\mathcal{S}(\mathbb{R})$ and have compact supports in $\mathbb{R} \backslash\{0\}$. By the Fubini theorem, the integral in the right-hand side of (5) is equal to

$$
\frac{i^{n-1}}{2} \int_{\mathbb{R}^{n}}\left(\sum_{\delta} \delta_{1} \cdot \ldots \cdot \delta_{n} \frac{\delta_{1} x_{1}+\cdots+\delta_{n} x_{n}}{x_{1} \ldots x_{n}} u\left(\delta_{1} x_{1}+\cdots+\delta_{n} x_{n}\right)\right) \phi(x) d x
$$

which completes the proof.

Putting $f(t)=|t|^{q}$ and $n=3$ in (2) we get the following fact which was used in [10] to prove that the space $\ell_{\infty}^{3}$ is not isometric to a subspace of $L_{q}$.

Corollary 1. For every $q>0$ which is not an even integer the Fourier transform of the function $\max ^{q}\left(\left|x_{1}\right|,\left|x_{2}\right|,\left|x_{3}\right|\right)$ is a continuous homogeneous sign-changing function on the set $G \subset \mathbb{R}^{3}$.

Proof. For every $q>0$ which is not an even integer, we have $\left(|t|^{q}\right)^{\wedge}(\xi)=c_{q}|\xi|^{-1-q}$, for all non-zero $\xi \in \mathbb{R}$. Apply the formula (2) with $f(t)=|t|^{q}$ and $n=3$ :

$$
\begin{aligned}
\left(\max ^{q}\left(\left|x_{1}\right|,\left|x_{2}\right|,\left|x_{3}\right|\right)\right)^{\wedge}\left(\xi_{1}, \xi_{2}, \xi_{3}\right)= & \frac{-c_{q}}{2 \xi_{1} \xi_{2} \xi_{3}}\left(\left|\xi_{1}+\xi_{2}+\xi_{3}\right|^{-q} \operatorname{sgn}\left(\xi_{1}+\xi_{2}+\xi_{3}\right)\right. \\
& -\left|\xi_{1}+\xi_{2}-\xi_{3}\right|^{-q} \operatorname{sgn}\left(\xi_{1}+\xi_{2}-\xi_{3}\right) \\
& -\left|\xi_{1}-\xi_{2}+\xi_{3}\right|^{-q} \operatorname{sgn}\left(\xi_{1}-\xi_{2}+\xi_{3}\right) \\
& \left.+\left|\xi_{1}-\xi_{2}-\xi_{3}\right|^{-q} \operatorname{sgn}\left(\xi_{1}-\xi_{2}-\xi_{3}\right)\right)
\end{aligned}
$$

for every $\xi=\left(\xi_{1}, \xi_{2}, \xi_{3}\right) \in G$. Clearly, the Fourier transform has opposite signs at the points $\xi_{1}=3, \xi_{2}=\xi_{3}=1$ and $\xi_{1}=3, \xi_{2}=\xi_{3}=2$.

\section{Isometric Embedding of Lorentz Sequence Spaces into $L_{q}$}

We are going to use the Fourier transform criterion mentioned in the Introduction to characterize the finite- and infinite-dimensional Lorentz sequence spaces which are isometric to subspaces of $L_{q}$. We formulated this criterion in the Introduction as a necessary condition for the existence of an isometric embedding. As a matter of fact, one can also use this criterion as a sufficient condition, although there may be some complications (for details, see [10, Remark 1]). For Banach spaces for which the distribution $\gamma$ is a finite measure one obtains the following Levy representation of the norm (named after Paul Levy):

$$
\|x\|^{q}=\int_{\mathbb{R}^{n-1}}\left|x_{1} \xi_{1}+\cdots+x_{n-1} \xi_{n-1}+x_{n}\right|^{q} d \gamma\left(\xi_{1}, \ldots, \xi_{n-1}\right) .
$$

It is easy to see [10] that the Levy representation with a finite measure $\gamma$ implies the existence of an isometric embedding of the space into $L_{q}$, and we shall use this fact in what follows. In particular, we shall use the Levy representation for the norm of the space $\ell_{\infty}^{2}$ [10]: for each $q<1$ and for all $x, y \in \mathbb{R}$,

$$
\max ^{q}(|x|,|y|)=\frac{1}{-} \cot (\pi q / 2) \int|x+y \xi|^{q} \underline{|\xi-1|^{-q}-|\xi+1|^{-q}} d \xi
$$


Note that $\gamma(\xi)=\frac{|\xi-1|^{-q}-|\xi+1|^{-q}}{\xi} d \xi$ is a finite measure on $\mathbb{R}$, which was calculated (using (2) above) in [10] as the Fourier transform of the function $\max ^{q}(|x|,|y|)$. If $q>1$ then the function $\gamma$ is still positive, but it is not integrable around 1 and -1 . Thus, the space $\ell_{\infty}^{2}$ is isometric to a subspace of $L_{q}$ if and only if $q \leq 1$. For $q=1$, the Levy representation is given by the well-known and particularly simple

$$
\max (|x|,|y|)=(1 / 2)(|x+y|+|x-y|) .
$$

Now we are ready to apply the Fourier transform criterion to Lorentz spaces.

Theorem 1. (a) Let $0<q \leq 1$. If $n \geq 2$ then the space $\ell_{w, q}^{n}$ is isometric to a subspace of $L_{q}$ if and only if

$$
a_{1}-a_{2}=a_{2}-a_{3}=\cdots=a_{n-1}-a_{n}
$$

(In particular, the space $\ell_{w, q}^{2}$ is isometric to a subspace of $L_{q}$ for every choice of $\left.a_{1}, a_{2} \cdot\right)$

(b) Let $q>1$ and $n \geq 2$. Then the space $\ell_{w, q}^{n}$ is isometric to a subspace of $L_{q}$ if and only if $a_{1}=\cdots=a_{n}$ (so that $\left.\ell_{w, q}^{n}=\ell_{q}^{n}\right)$.

Proof. Suppose that $n \geq 2$ and that the space $\ell_{w, q}^{n}$ is isometric to a subspace of $L_{q}$. Let us prove (8) by induction. The hyperplane in $\ell_{w, q}^{n}$ defined by $x_{n}=0$ is isometric to the $(n-1)$-dimensional Lorentz space with the weights $a_{1}, \ldots, a_{n-1}$. By the induction hypothesis, $a_{1}-a_{2}=a_{2}-a_{3}=\cdots=a_{n-2}-a_{n-1}=\alpha$. Denote $a_{n-1}-a_{n}$ by $\beta$.

For every $k \geq 3$, we have

$$
\sum_{j=1}^{k}(-1)^{j-1}\left(\begin{array}{c}
k-1 \\
j-1
\end{array}\right) a_{n-k+j}=(-1)^{k}(\beta-\alpha) .
$$

To see this, let $a_{n-k+1}=u$; then $a_{n-k+j}=u-(j-1) \alpha$ for $j=1, \ldots, k-1$, and $a_{n}=u-(k-2) \alpha-\beta$. So the sum in (9) is equal to

$$
u \sum_{j=1}^{k}(-1)^{j-1}\left(\begin{array}{c}
k-1 \\
j-1
\end{array}\right)+(-1)^{k} \beta+\sum_{j=1}^{k}(-1)^{j}(j-1)\left(\begin{array}{c}
k-1 \\
j-1
\end{array}\right) \alpha-(-1)^{k} \alpha
$$

and it suffices to note that $\sum_{j=1}^{k}(-1)^{j-1}\left(\begin{array}{c}k-1 \\ j-1\end{array}\right)=0$ and $\sum_{j=1}^{k}(-1)^{j}(j-1)\left(\begin{array}{l}k-1 \\ j-1\end{array}\right)=0$.

Apply (1) with $f(t)=|t|^{q}$ and use (9) to get the following expression for the norm of the space $\ell_{w, q}^{n}$ :

$$
\begin{aligned}
\|x\|^{q}=a_{1}\left(x_{1}^{*}\right)^{q}+\cdots+a_{n}\left(x_{n}^{*}\right)^{q}= & a_{n}\left(\left|x_{1}\right|^{q}+\cdots+\left|x_{n}\right|^{q}\right)+\beta \sum_{i<j} \max ^{q}\left(\left|x_{i}\right|,\left|x_{j}\right|\right) \\
& +(\beta-\alpha) \sum^{n}(-1)^{k} \sum \max ^{q}\left(\left|x_{i_{1}}\right|, \ldots,\left|x_{i_{k}}\right|\right) .
\end{aligned}
$$


Consider the three-dimensional subspace of $\ell_{w, q}^{n}$ consisting of the vectors $x \in \mathbb{R}^{n}$ for which $x_{3}=\cdots=x_{n}$. It follows from (10) that this subspace is isometric to a space whose norm can be represented in the form

$$
\left\|\left(x_{1}, x_{2}, x_{3}\right)\right\|^{q}=(\beta-\alpha) \sum_{k=3}^{n}(-1)^{k}\left(\begin{array}{l}
n-2 \\
k-2
\end{array}\right) \max ^{q}\left(\left|x_{1}\right|,\left|x_{2}\right|,\left|x_{3}\right|\right)+g\left(x_{1}, x_{2}, x_{3}\right),
$$

where $g$ is a linear combination of the functions $\left|x_{i}\right|^{q}$ and $\max ^{q}\left(\left|x_{i}\right|,\left|x_{j}\right|\right)$ and, therefore, the Fourier transform of $g$ is supported in the coordinate planes in $\mathbb{R}^{3}$. Note that $\sum_{k=3}^{n}(-1)^{k}\left(\begin{array}{l}n-2 \\ k-2\end{array}\right)=-1$, and so

$$
\left\|\left(x_{1}, x_{2}, x_{3}\right)\right\|^{q}=(\alpha-\beta) \max ^{q}\left(\left|x_{1}\right|,\left|x_{2}\right|,\left|x_{3}\right|\right)+g\left(x_{1}, x_{2}, x_{3}\right) .
$$

This three-dimensional space will be isometric to a subspace of $L_{q}$. By the Fourier transform criterion discussed above, the distribution

$$
\gamma\left(\xi_{1}, \xi_{2}\right)=\left(1 /\left((2 \pi)^{2} c_{q}\right)\right)\left(\left\|\left(x_{1}, x_{2}, x_{3}\right)\right\|^{q}\right)^{\wedge}\left(\xi_{1}, \xi_{2}, 1\right)
$$

will be a finite measure in $\mathbb{R}^{2}$ (and, in particular, it must be non-negative). On the other hand, by Corollary 1 , the Fourier transform of $\max ^{q}\left(\left|x_{1}\right|,\left|x_{2}\right|,\left|x_{3}\right|\right)$ is a continuous homogeneous function which changes its sign on an open set $G \subset \mathbb{R}^{3}$, and so $\gamma$ cannot be non-negative if $\alpha \neq \beta$. Thus, $\alpha=\beta$, which concludes the proof of $(8)$.

Conversely, suppose that (8) is satisfied. Then, for $k \geq 3$, all of the coefficients $\sum_{j=1}^{k}(-1)^{j-1}\left(\begin{array}{c}k-1 \\ j-1\end{array}\right) a_{n-k+j}$ in (1) are equal to zero, and the formula (1) turns into

$$
\begin{aligned}
\|x\|^{q} & =a_{1}\left(x_{1}^{*}\right)^{q}+\cdots+a_{n}\left(x_{n}^{*}\right)^{q} \\
& =a_{n}\left(\left|x_{1}\right|^{q}+\cdots+\left|x_{n}\right|^{q}\right)+\left(a_{n-1}-a_{n}\right) \sum_{i<j} \max ^{q}\left(\left|x_{i}\right|,\left|x_{j}\right|\right) .
\end{aligned}
$$

If $q<1$, then the norm admits the Levy representation with a finite measure which is the sum of the measures appearing in (6) and of point-masses $a_{n}$ at the points $(1,0, \ldots, 0), \ldots,(0, \ldots, 0,1)$. So the space $\ell_{w, q}^{n}$ is isometric to a subspace of $L_{q}$. In the case $q=1$ one easily gets the Levy representation using (7). This finishes the proof of part (a).

If $q>1$, and if $q$ is not an even integer, then by the remark at the beginning of Section 3, the Fourier transforms of the functions $\max ^{q}\left(\left|x_{i}\right|,\left|x_{j}\right|\right)$ are not finite measures. So the space $\ell_{w, q}^{n}$ can be isometric to a subspace of $L_{q}$ only if all these functions are missing in (11), which happens if and only if $a_{n-1}=a_{n}$. In conjunction with (8) this gives $a_{1}=\cdots=a_{n}$, and we have proved part (b). (The case where $q$ is an even integer will be dealt with below.)

The Fourier transform criterion does not work in the case where $q$ is an even integer. However, statement (b) of Theorem 1 remains true in this case. The following simple fact shows that, for every $q>1$, the space $\ell_{w, q}^{n}$ is smooth only if all the numbers $a_{k}$ are equal. (Thus, for $q>1$, the space $\ell_{w, q}^{n}$ is isometric to a 
Lemma 2. For every $q>1$, the space $\ell_{w, q}^{n}$ is smooth if and only if $a_{1}=\cdots=a_{n}$. Proof. Consider the two-dimensional subspace of $\ell_{w, q}^{n}$ consisting of the vectors $x=\left(x_{1}, \ldots, x_{n}\right)$ for which $x_{1}=\cdots=x_{n-1}$. This subspace is isometric to the two-dimensional Banach space whose norm is given by

$$
\|(x, y)\|^{q}= \begin{cases}\left(a_{1}+\cdots+a_{n-1}\right)|x|^{q}+a_{n}|y|^{q} & \text { for }|x| \geq|y| \\ \left(a_{2}+\cdots+a_{n}\right)|x|^{q}+a_{1}|y|^{q} & \text { for }|x| \leq|y| .\end{cases}
$$

Let $a=a_{1}+\cdots+a_{n-1}, b=a_{n}, c=a_{2}+\cdots+a_{n}$, and $d=a_{1}$. The curves $a|x|^{q}+b|y|^{q}=1$ and $c|x|^{q}+d|y|^{q}=1$ intersect at the point whose $x$-coordinate equals $(1 /(a+b))^{1 / q}$. (Note that $a+b=c+d$.) If the space is smooth then the derivatives of the functions $y=\left(\frac{1-a x^{q}}{b}\right)^{1 / q}$ and $y=\left(\frac{1-c x^{q}}{d}\right)^{1 / q}$ at the point $x=(1 /(a+b))^{1 / q}=(1 /(c+d))^{1 / q}$ must agree. This gives

$$
\left(\frac{1-a x^{q}}{b}\right)^{\frac{1}{q}-1} \frac{a x^{q-1}}{b}=\left(\frac{1-c x^{q}}{d}\right)^{\frac{1}{q}-1} \frac{c x^{q-1}}{d}
$$

and, therefore, $a / b=c / d$. This means that $a_{1}=a_{n}$, and we are done.

Because a decreasing arithmetic progression of positive numbers is either constant or finite, we immediately deduce from Theorem 1 the following corollary.

Corollary 2. Let $0<q<\infty$. The Lorentz sequence space $\ell_{w, q}$ is isometric to a subspace of $L_{q}$ if and only if $w$ is a constant sequence.

A geometrical argument. For $q=1$, we should like to sketch a simple geometrical proof of Theorem 1. First we prove by induction that $\ell_{w, 1}^{n}$ is isometric to a subspace of $L_{1}$ only if the weights $a_{k}$ satisfy (8). Let $E$ be a Lorentz space of dimension $n+1$ with weights $a_{1}, \ldots, a_{n+1}$. If $E$ is isometric to a subspace of $L_{1}$ then (8) holds by hypothesis, and so it suffices to prove that $a_{n-1}-a_{n}=a_{n}-a_{n+1}$ to complete the induction. In particular, we may assume that $a_{n-1}, a_{n}$ and $a_{n+1}$ are not all equal. Let $B^{*}$ denote the unit ball of $E^{*}$. Since the unit ball of $E$ is a polytope, and since $E$ is isometric to a subspace of $L_{1}$, it follows (see e.g. [1]) that $B^{*}$ is a zonotope (that is, a Minkowski sum of line segments). By [1, Theorem 3.3], all of the two-dimensional faces of $B^{*}$ are centrally symmetric. It is easily seen that the extreme points of $B^{*}$ are all the sign-changed permutations of the vector $\mathbf{a}=\left(a_{1}, a_{2}, \ldots, a_{n+1}\right)$. In particular, one of the two-dimensional faces of $B^{*}$ has as its vertices all of the vectors obtained by permuting the last three coordinates of a. If $a_{n-1}=a_{n}$ or if $a_{n}=a_{n+1}$, then this face is triangular, which contradicts the central symmetry requirement. If $a_{n-1}>a_{n}>a_{n+1}$, then the face is hexagonal, and the symmetry condition forces $a_{n-1}-a_{n}=a_{n}-a_{n+1}$ as required. Thus (8) is a necessary condition. To show that (8) is also sufficient, one can check that if (8) is satisfied then $B^{*}$ has four kinds of two-dimensional faces: two classes of quadrilateral faces, one class of octagonal faces, and one class of hexagonal faces like 
symmetric without any condition on the weights, while (8) guarantees that the hexagonal faces are also centrally symmetric. So, by [1, Theorem 3.3] once again, if (8) is satisfied, then $B^{*}$ is a zonotope, and hence $E$ is isometric to a subspace of $L_{1}$.

\section{Isometric Embedding of Lorentz Function Spaces into $L_{q}$}

For $0 \leq \alpha \leq 2$, let $w_{\alpha}(t)=1+\frac{\alpha}{2}-\alpha t$. Observe that the $w_{\alpha}$ 's are precisely the decreasing linear weights on $[0,1]$ which satisfy $\int_{0}^{1} w(t) d t=1$.

Theorem 2. (a) Let $0<q \leq 1$. Then $L_{w, q}(0,1)$ is isometric to a subspace of $L_{q}$ if and only if $w=w_{\alpha}$ for some $\alpha \in[0,2]$.

(b) Let $q>1$. Then $L_{w, q}(0,1)$ is isometric to a subspace of $L_{q}$ if and only if $w=w_{0}$ (so that $L_{w, q}=L_{q}$ ).

Proof. (a) First we prove necessity. For $n \geq 1$ and for $1 \leq k \leq n$, let $e_{n, k}=$ $\chi_{\left[\frac{k-1}{n}, \frac{k}{n}\right)}$, and let $X_{n}$ be the linear span of the $e_{n, k}$ 's in $L_{w, q}$. Clearly, $X_{n}$ is isometric to a Lorentz space with weights $a_{n, k}=\int_{(k-1) / n}^{k / n} w(t) d t$. By Theorem 1 , the sequence $\left\langle a_{n, k}\right\rangle_{k=1}^{n}$ forms a decreasing arithmetic progression for each $n$. This clearly forces $w$ to be a decreasing linear function, so that $w=w_{\alpha}$ for some $\alpha \in[0,2]$. For sufficiency, observe that if $w=w_{\alpha}$, then by Theorem 1 each $X_{n}$ is isometric to a subspace of $L_{q}$. Since $\cup_{n \geq 1} X_{n}$ is dense in $L_{w, q}$, it follows from the fact that the $L_{q}$ spaces are stable under the operation of taking ultrapowers that $L_{w, q}$ is isometric to a subspace of $L_{q}$ (e.g. [14, pp. 121-122]).

(b) In this case, by Theorem $1,\left\langle a_{n, k}\right\rangle_{k=1}^{n}$ is a constant sequence for each $n$, which forces $w$ to be constant. Sufficiency is obvious in this case.

For the function spaces $L_{w, q}(0, \infty)$ the situation is different. Arguing as above and as in Corollary 2 one immediately obtains the following corollary.

Corollary 3. Let $0<q<\infty$. Then $L_{w, q}(0, \infty)$ is isometric to a subspace of $L_{q}$ if and only if $w(t)$ is a constant function.

Remarks. 1. Clearly, the $L_{w_{\alpha}, 1}$ spaces are all isomorphic to $L_{1}$ (in fact, the $L_{w_{\alpha}, 1}$ and $L_{1}$ norms are equivalent). On the other hand, the proof of a result of Carothers, Dilworth and Trautman [5, Theorem 2.3] shows that there is no isometry from $L_{w_{\alpha}, 1}$ onto $L_{w_{\beta}, 1}$ if $0 \leq \alpha<\beta \leq 2$.

2. For $\alpha>0$, there is no isometry from $L_{1}$ into $L_{w_{\alpha}, 1}$. In fact, by [5, Lemma 2.1] there is not even an isometry from $\ell_{1}^{2}$ into $L_{w_{\alpha}, 1}$.

3. Observe that $L_{w_{2}, 1}$ has a certain universality property among the $L_{w_{\alpha}, 1}$ spaces. To be precise, if $0<\alpha \leq 2$, then $L_{w_{\alpha}, 1}$ is isometric by a dilation to the onecomplemented sublattice of $L_{w_{2}, 1}$ which consists of all functions that are supported in the interval $\left[0, \frac{2 \alpha}{\alpha+2}\right]$. Moreover, $L_{w_{2}, 1}$ contains a continuum of isometrically distinct one-complemented (by conditional expectation) two-dimensional subspaces: 
balls are octagonal). This is in contrast with $L_{1}$, which has, for each positive integer $n$, a unique one-complemented $n$-dimensional subspace up to isometry, namely $\ell_{1}^{n}$. 4. Let us recall some of what is known about the geometry of the $L_{w, 1}$ spaces. For every strictly decreasing $w$, the extreme points of the unit ball of $L_{w, 1}$ are all the functions of the form $\varepsilon \chi_{A} /\left(\int_{0}^{|A|} w(t) d t\right)$, where $A$ is any subset of $[0,1]$ with positive Lebesgue measure $|A|$, and $\varepsilon$ is any \pm 1 -valued measurable function ([5, Prop. $2.2]$ ). Moreover, while $L_{w, 1}$ is not strictly convex, it is nevertheless true that every element on the unit sphere of $L_{w, 1}$ is the barycenter of a unique Borel probability measure supported on the extreme points of the ball ([5, Theorem 3.5]). Finally, Sedaev [20] proved that if $w$ is strictly decreasing then $L_{w, 1}$ has the Kadec-Klee property, i.e., if $f_{n} \rightarrow f$ weakly and $\left\|f_{n}\right\|_{w, 1} \rightarrow\|f\|_{w, 1}$ then $\left\|f-f_{n}\right\|_{w, 1} \rightarrow 0$. It is well-known, on the other hand, that $L_{1}$ does not have this property.

\section{Positive Definite Functions}

We would like to mention how the problems considered in this paper are connected to positive definite functions. The question we wish to discuss is as follows: for which $q \in(0,2]$ and for which $a_{1}, \ldots, a_{n} \geq 0$ is the function $\exp \left(-a_{1}\left(x_{1}^{*}\right)^{q}-\right.$ $\cdots-a_{n}\left(x_{n}^{*}\right)^{q}$ ) positive definite on $\mathbb{R}^{n}$ ? (Note that, for every $q>2$, this function is not positive definite because its one-dimensional restriction $\exp \left(-|t|^{q}\right)(t \in \mathbb{R})$ is not positive definite for these values of $q$.)

If $a_{1}=1$ and $a_{2}=\cdots=a_{n}=0$ we arrive at the following question: for which $q$ is the function $\exp \left(-\max ^{q}\left(\left|x_{1}\right|, \ldots,\left|x_{n}\right|\right)\right)$ positive definite? This is exactly the problem posed in 1938 by I. J. Schoenberg [18] and solved by J. Misiewicz [16] in 1989. The answer is that, for every $n \geq 3$ and $q>0$, the function is not positive definite, and, for $n=2$, the function is positive definite if and only if $q \leq 1$. The problems of Schoenberg's type are important in the study of isotropic and stable random vectors (for details, see [10]).

The connection between positive definite functions and isometric embeddings into $L_{q}$ was discovered in 1966 by J. Bretagnolle, D. Dacunha-Castelle and J. L. Krivine [2]: for $q \in(0,2]$, a Banach space $(E,\|\cdot\|)$ is isometric to a subspace of $L_{q}$ if and only if the function $\exp \left(-\|x\|^{q}\right)$ is positive definite.

Combining this result with Theorem 1 we get an answer to the question raised above.

Proposition 3. The function $\exp \left(-a_{1}\left(x_{1}^{*}\right)^{q}-\cdots-a_{n}\left(x_{n}^{*}\right)^{q}\right)$ is positive definite if and only if : (i) $q \in(1,2]$ and $a_{1}=\cdots=a_{n}$, or

(ii) $q \leq 1$ and the numbers $a_{k}$ form a non-increasing arithmetic progression. (This includes the case $n=2$ where the progression consists of two numbers only.)

Since we no longer assume in the formulation of Proposition 3 that $a_{1} \geq \cdots \geq a_{n}$ (which was necessary in the definition of the Lorentz spaces) we have to make a remark concerning the proof. Formally, without the condition $a_{1} \geq \cdots \geq a_{n}$ 
cannot apply the result from [2] directly. We can, however, use a generalization of this result from [17, p.290] stating that, for any continuous one-homogeneous non-negative function $u$ on $\mathbb{R}^{n}$ which vanishes only at the origin, if $\exp \left(-u(x)^{q}\right)$ is positive definite, then $u$ is the norm (or $q$-norm if $q<1$ ) of a subspace of $L_{q}$. Besides, the proof of Theorem 1 does not depend on the condition $a_{1} \geq \cdots \geq a_{n}$, and, in fact, in the proof of Theorem 1 this condition follows from the existence of an isometric embedding into $L_{q}$.

\section{REFERENCES}

1. E. D. Bolker, A class of convex bodies, Trans. Amer. Math. Soc. Vol 145 (1969), 323-345.

2. J. Bretagnolle, D. Dacunha-Castelle and J. L. Krivine, Lois stables et espaces $L_{p}$, Ann. Inst. H. Poincaré Probab. Statist. Vol 2 (1966), 231-259.

3. N. L. Carothers and S. J. Dilworth, Subspaces of $L_{p, q}$, Proc. Amer. Math. Soc. Vol 104 (1988), $537-545$.

4. N. L. Carothers and S. J. Dilworth, Some Banach space embeddings of classical function spaces, Bull. Austral. Math. Soc. Vol 43 (1991), 73-77.

5. N. L. Carothers, S. J. Dilworth and D. A. Trautman, On the geometry of the unit spheres of the Lorentz spaces $L_{w, 1}$, Glasgow Math. J. Vol 34 (1992), 21-25.

6. N. L. Carothers and B. Turett, Isometries on $L_{p, 1}$, Trans. Amer. Math. Soc. Vol 297 (1986), 85-103.

7. N. L. Carothers, R. G. Haydon and Pei-Kee Lin, On the isometries of the Lorentz function spaces, Israel J. Math. Vol 84 (1993), 265-287.

8. T. Figiel, W. B. Johnson and L. Tzafriri, On Banach lattices and spaces having local unconditional structure with applications to Lorentz function spaces, J. Approx. Theory Vol 13 (1975), 297-312.

9. I. M. Gelfand, M. I. Graev and N. Y. Vilenkin, Generalized functions 5, Academic Press, New York, 1966.

10. A. L. Koldobsky, Generalized Levy representation of norms and isometric embeddings into $L_{p}$-spaces, Ann. Inst. H. Poincaré Probab. Statist. Vol 28 (1992), 335-353.

11. A. L. Koldobsky, Characterization of measures by potentials, J. Theoret. Probab. Vol 7 (1994), 135-145.

12. Mireille Levy, L'espace d'interpolation réel $\left(A_{0}, A_{1}\right)_{\theta, p}$ contient $l^{p}$, C. R. Acad. Sci. Paris Sér. A-B Vol 289 (1979), A675-A677.

13. Mireille Levy, Structure fine des espaces d'interpolation réels; applications aux espaces de Lorentz, Thesis, Université de Paris 6, Paris, 1980.

14. J. Lindenstrauss and L. Tzafriri, Classical Banach spaces, Lecture Notes in Math. Vol. 338, Springer-Verlag, Berlin-Heidelberg-New York, 1973.

15. G. G. Lorentz, Some new functional spaces, Ann. of Math. Vol 51 (1950), 37-55.

16. J. Misiewicz, Positive definite functions on $\ell_{\infty}$, Statist. Probab. Lett. Vol 8 (1989), 255-260.

17. J. Misiewicz and Cz. Ryll-Nardzewski, Norm dependent positive definite functions, Lecture Notes in Math. Vol 1391 (1987), 284-292.

18. I. J. Schoenberg, Metric spaces and positive definite functions, Trans. Amer. Math. Soc. Vol 44 (1938), 522-536.

19. C. Schütt, Lorentz spaces which are isomorphic to subspaces of $L^{1}$, Trans. Amer. Math. Soc. Vol 314 (1989), 583-595.

20. A. A. Sedaev, The H-property in symmetric spaces, (Russian), Teor. Funktsĭ Funktsional. Anal. i Prilozhen. Vol 11 (1970), 67-80.

Department of Mathematics, University of South Carolina, Columbia, SC 29208, U.S.A.

Current address: Department of Mathematics, Texas A\&M University, College Station, Texas 77843, U.S.A.

E-mail address: dilworth@math.scarolina.edu

Division of Mathematics, Computer Science, and Statistics, University of Texas 
THE FOURIER TRANSFORM OF ORDER STATISTICS AND LORENTZ SPACES 13

E-mail address: koldobsk@ringer.cs.utsa.edu 